\title{
Development of elastically graded titanium alloys for biomedical applications
}

\author{
Stéphanie DELANNOY ${ }^{\mathrm{a}, \mathrm{b}^{*}}$, Sarah BAÏZ ${ }^{\mathrm{c}}$, Pascal LAHEURTE ${ }^{\mathrm{d}}$, Laurence JORDAN ${ }^{\mathrm{a}, e, f}$, Frédéric PRIMA $^{\mathrm{a}}$ \\ a PSL Research University, Chimie ParisTech - CNRS, Institut de Recherche de Chimie Paris UMR CNRS 8247, \\ 75005 Paris, France
}

b BIOTECH DENTAL, 13300 Salon-de-Provence, France

${ }^{\mathrm{c}}$ Laboratoire Procédés et Ingénierie en Mécanique et Matériaux, PIMM, ENSAM, UMR 8006, CNRS, CNAM, 75013 Paris, France

d Laboratoire d'Etude des Microstructures et de Mécanique des Matériaux, LEM3 UMR CNRS 7239, Université de Lorraine, île du Saulcy, 57045 Metz Cedex, France

e Paris Diderot University, Sorbonne Paris Cité, Faculty of dentistry, 75013 Paris, France

${ }^{\mathrm{f}}$ Hospital Rothschild, AP-HP, 75012 Paris, France

*corresponding author: stephanie.delannoy@chimieparistech.psl.eu

\begin{abstract}
Recent works have shown that the elastic mismatch observed at the bone / implant interface could be responsible for stress shielding issues causing bone resorption phenomena and potentially implant failures. In the present study, new advanced thermomechanical approaches leading to titanium alloys with graded elastic properties are proposed. The underlying philosophy and the whole methodology is detailed here, from the selection of candidates with large elastic variability to the creation of gradients, involving the identification of microstructure-properties relationships and the use of appropriate thermo-mechanical treatments. Applied on Ti-Nb-Zr alloys, these original routes enabled to get the following graded properties: elastic modulus from 85 to $65 \mathrm{GPa}$ over $400 \mu \mathrm{m}$ for TNZ alloy by surface deformation, and from 130 to $75 \mathrm{GPa}$ over $100 \mu \mathrm{m}$ for Ti-13-13 by preferential dissolution. These promising results thus validated the previously designed material-strategy-process combinations.
\end{abstract}

\section{Introduction}

The notion of biocompatibility surrounding implanted medical devices has continued to evolve over time, and the technological advances, as well as a better understanding of the human body, shifted the model from being based on the material itself to being based on the device and its application [1]. Therefore biocompatibility is no longer solely perceived as bio-inertness, and efforts are made to reach an active biocompatibility where the host response is crucial.

In the field of dental implantology the aim is to promote bone remodeling around the implant to ensure good osseointegration. To achieve this, in addition to biological and chemical sides, mechanical aspects has to be taken into special consideration in the development of biomaterials. Indeed, at the start of the physiological process, mechanical stimuli help regulate the osseous synthesis. To get a suitable density, it is thus necessary to maintain the load applied on the bone within a certain range of stress, outside of which resorption or necrosis could arise [2], [3]. Unfortunately, use of dental implant generates changes that can easily disturb this equilibrium. First, it results in the loss of the periodontal ligament, a biological element which provides a damping effect ensuring an adapted load transfer to the surrounding bone [4], [5]. Moreover, the mismatch in the Young's modulus (E) observed at the bone / implant interface is reported to be involved in stress shielding issues, leading to a poor stress distribution [6], [7]. In fact, although much lower than those of materials used in the past (as 316L steel or Co-Cr-Mo), elastic moduli of CP Ti or TA6V, the main titanium alloys currently used, remain almost 5 times that of bone. This project was then initiated to improve this axis.

Several research works have been undertaken to lower the modulus of titanium alloys. The increase in the porosity [8]-[11] or the application of low-modulus coatings [12] enabled to significantly reduce the stiffness of the studied materials, but at the expense of their other mechanical properties. As a matter of fact, in addition to difficulties in process control, the previously mentioned technical solutions can cause a drop in mechanical resistance [13], [14] or a weakening owing to the multi-material interfaces [15]. In this context, the new generation of low-modulus titanium alloys, which began developing in the 90's and can 
reach values close to $60 \mathrm{GPa}$, still seems to offer the best combination of properties today [2], [16]. Low stiffness in the whole implant could however compromise its stability and its durability. Since iso-elasticity is essentially required at the interface with bone, the original idea we chose to explore consists in using the "low-modulus" feature of this kind of alloys only on the implants surface, and to produce a stiffer state in the core material. In other words, this project concerns the development of titanium alloys displaying elasticity gradient resulting from microstructural gradient, thus preserving a monolithic material with a unique nominal chemical composition [17].

The different steps of the methodology adopted to implement this concept will be presented in this article. Experimental details and results can be found inside the different parts.

\section{$\underline{\text { Selection of candidate materials }}$}

Titanium alloys can display a wide variety of microstructures and properties according to their composition or their thermomechanical treatment. Two axes have guided the selection of candidate materials.

First, since a final use in human body is concerned, alloying elements have been limited to those presenting a proven harmlessness, eliminating, in particular, species such as $\mathrm{Al}$ or $\mathrm{V}$, nevertheless used in TA6V alloy, but becoming increasingly controversial as a result of potentially inducing cytotoxicity or neurodegenerative diseases [18]-[21]. Among highly biocompatible elements $\mathrm{Pt}, \mathrm{Ta}, \mathrm{Nb}$ and $\mathrm{Zr}$ [19], mainly for economic purpose, we opted for the Ti-Nb-Zr family.

Thereafter, efforts were made to refine our choice among this family, with the will, this time, to reach our mechanical specifications, namely by selecting alloys able to create different structures with large gap in modulus. We have then followed a design method based on the use of parameters which can predict the properties of alloys. Indeed, the tendency of titanium alloys to display a low modulus and, especially, a great elastic variability, is related to their instability. And the latter can be estimated using different key factors. For example, an electronic approach developed by Morinaga is based on two parameters, Bo (bond order) and $\mathrm{Md}$ (d-orbital energy), calculated from clusters representative of the crystalline structure [22]. The analysis of the Bo-Md diagrams resulting from this method enables to highlight areas, and thus range of composition, of low-modulus alloys associated with an instability of the $\beta$ phase, involving thermally or mechanically triggered martensitic transformation [23]. Combined with studies of structural predictions using e/a parameter (electron to atom ratio) [24]-[27], and with data from the literature about $\mathrm{Nb}$ and Zr-containing titanium alloys [2], [28]-[33], these considerations led to the choice of two materials which are presented in Table 1.

\begin{tabular}{|c|c|c|c|c|c|c|c|c|c|c|}
\hline \multirow{3}{*}{ Alloy designation } & \multicolumn{6}{|c|}{ Composition } & \multirow{2}{*}{\multicolumn{4}{|c|}{ Indicators of $\beta$ phase stability }} \\
\hline & \multicolumn{3}{|c|}{$w t \%$} & \multicolumn{3}{|c|}{ at \% } & & & & \\
\hline & $\mathbf{T i}$ & $\mathbf{N b}$ & $\mathbf{Z r}$ & $\mathbf{T i}$ & $\mathbf{N b}$ & $\mathbf{Z r}$ & e/a & $\overline{\mathbf{B o}}$ & $\overline{\mathbf{M d}}$ & Moeq \\
\hline $\begin{array}{c}\text { Ti-13-13 } \\
\text { Ti-13Nb-13Zr (wt } \%)\end{array}$ & 74 & 13 & 13 & 84.56 & 7.65 & 7.79 & 4.08 & 2.84 & 2.48 & 3.64 \\
\hline $\begin{array}{c}\text { TNZ } \\
\text { Ti-20Nb-6Zr (at } \%) \\
\end{array}$ & 59.56 & 31.24 & 9.20 & 74 & 20 & 6 & 4.20 & 2.87 & 2.47 & 8.74 \\
\hline
\end{tabular}

Table 1 - Compositions and stability indicators of the chosen alloys: Ti-13-13 and TNZ.

Ti-13-13 can be classified as an $\alpha+\beta$ alloy, being fully martensitic when quenched from the $\beta$ field. It was developed for orthopaedic implantology in the 90's and was studied quite a lot since [34]-[37]. TNZ is, for its part, a metastable $\beta$ alloy, whose composition allows $\beta$ phase to be retained at room temperature, but in a metastable form which could further transform into martensite under mechanical stress. This alloy was designed to couple superelasticity and apparent low modulus [33], [38].

\section{Assessment of the elastic variability of the alloys}

Following alloys selection step, practical work was carried out to verify experimentally their elastic variability and determine their elastic range, associated microstructures and thermo-mechanical treatments used to get them. The aim here is not to create a gradient but to estimate the boundary properties of the materials. To facilitate the experiments and characterizations, flat thin samples were therefore used.

Parts of Ti-13-13 and TNZ bars were first solution treated under air at $1173 \mathrm{~K}$ during 15 min and water quenched. Outer oxidized part was removed by mechanical polishing and pieces were then cold-rolled (CR) at room temperature down to about $0.5 \mathrm{~mm}$ of thickness (reduction ratio $\approx 90 \%$ ). From this initial state, some samples were subjected to various additional thermal 
treatments under controlled atmosphere (argon). To measure mechanical properties, and particularly elastic modulus, flat tensile specimens were taken along the rolling direction and tested at a strain rate of $10^{-3} \mathrm{~s}^{-1}$ on an INSTRON 5966 machine equipped with a $10 \mathrm{~mm}$ gauge length extensometer. Phase constitution was determined by X-ray diffraction (XRD) using $\mathrm{Cu}$-K $\alpha$ radiation and microstructure was characterized by SEM (Zeiss LEO-1530) and TEM (JEOL 2000FX). Samples were respectively prepared by mechanical and electrochemical twin-jet polishing; the latter using a solution of methanol, 2-butoxyethanol, perchloric and hydrochloric acids around $260 \mathrm{~K}$.

Among numerous tested conditions, as it can be seen from Figure 1 and Figure 2, three interesting states were identified for each alloy. In both cases, $\mathrm{E}^{-}$corresponds to a low-modulus state obtained after quenching from the $\beta$ field. Deforming this state enabled to lower the modulus even more $\left(\mathrm{E}^{-}-\right)$, by rearrangement of variants of the pre-existing martensite (Ti-13-13) or by martensitic transformation of the $\beta$ phase (TNZ). As for the high-modulus state $\left(\mathrm{E}^{+}\right)$, it resulted from the precipitation of another phase at a fine scale, $\alpha$ for Ti-13-13 and $\omega$ for TNZ.

It is also interesting to note that the levels of mechanical resistance which have been observed for these states are satisfying and suit the targeted application (ultimate tensile strength $>700 \mathrm{MPa}$ for all, except for quenched TNZ which is about $500 \mathrm{MPa}$ ). Moreover, the lower elastic bounds are very low, around 50GPa, which is really promising to reduce the elastic discontinuity at the bone / implant interface. Since the elastic variability has been verified, strategies can be developed to produce graded pieces.

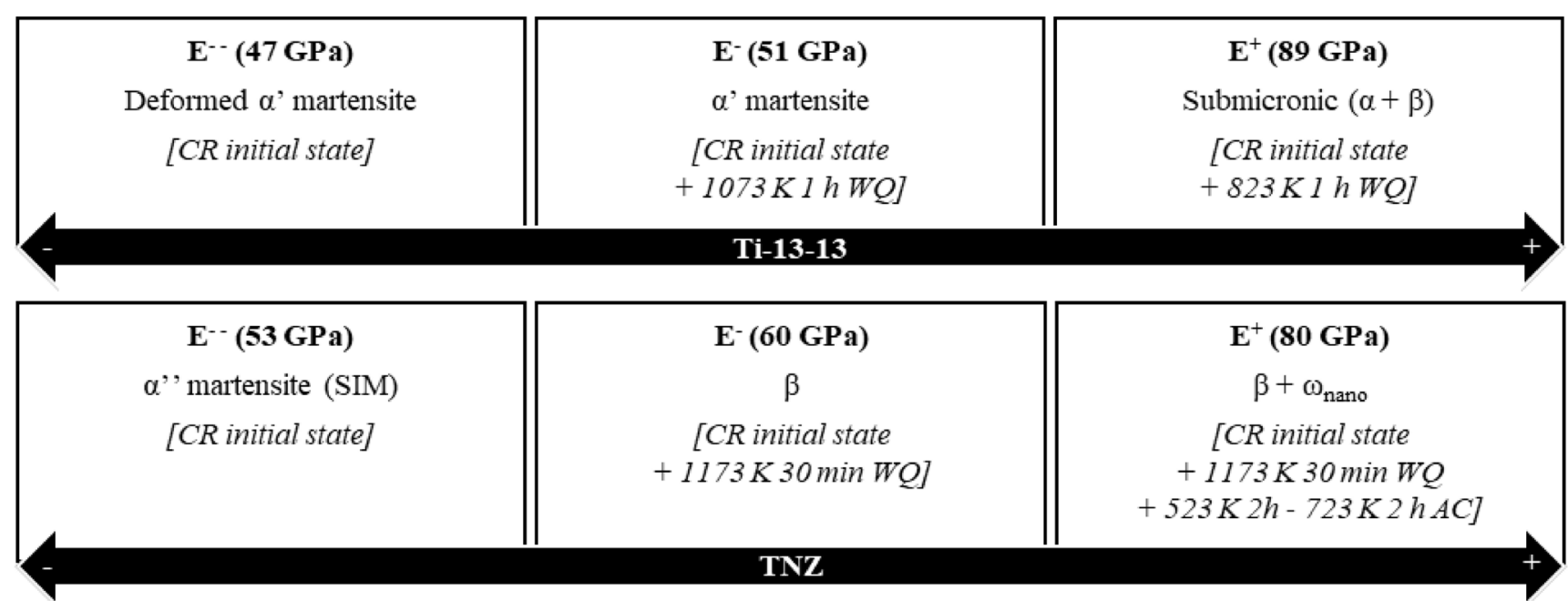

Figure 1 - Accessible elastic range of Ti-13-13 and TNZ alloys, associated microstructures and thermo-mechanical treatments.
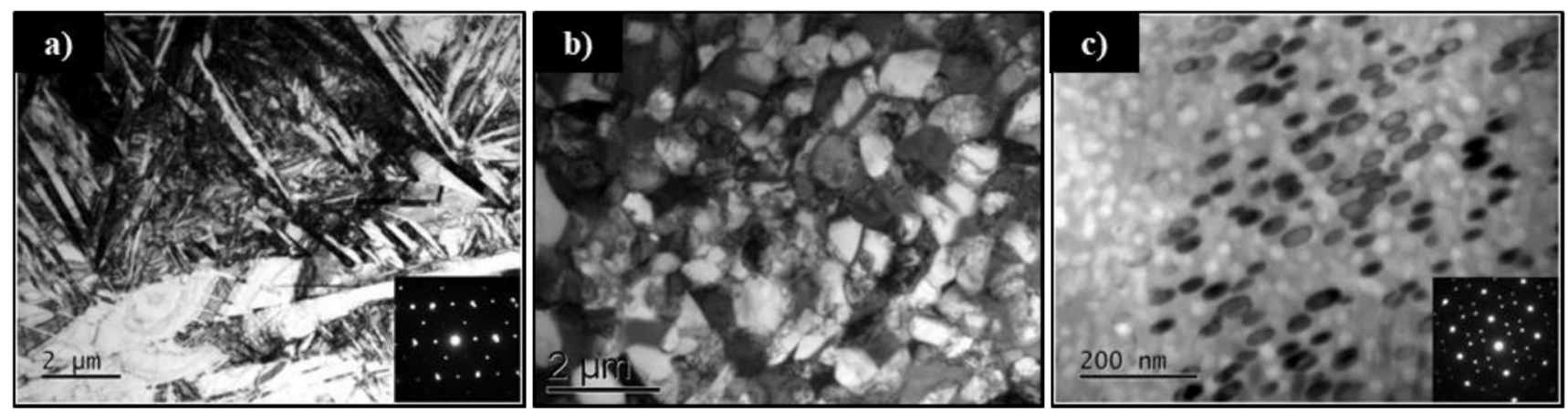

Figure 2 - Microstructural observations by TEM of Ti-13-13, (a) $\mathbf{E}^{-}$state and (b) $\mathbf{E}^{+}$state, and TNZ (c) $\mathbf{E}^{+}$state. Precisions about the states can be found in figure 1 .

\section{Creation of elastically graded materials}

Based on the previous results, two appropriate strategies involving mechanical deformation and thermal treatments were established, and each was applied on one of the alloys to create elasticity gradients.

The method used to get the initial state was the same as the one described for flat samples, except pieces were this time thicker at the beginning and cold-rolled down to about $5 \mathrm{~mm}$ in thickness (reduction ratio $\approx 70 \%$ ). Small plates, $2 \mathrm{~cm} \times 1 \mathrm{~cm}$, were then prepared. At the end of the specific treatments, samples were cut in half, mounted in resin and mirror polished to perform elasticity modulus measurements by instrumented indentation. Conditions used on the Anton Paar Micro Hardness Tester (ENSAM, Paris) were the following ones: Vickers indent, $500 \mathrm{mN}$ load with $5 \mathrm{~s}$ holding. It is worth noting that indentation modulus values can often 
be slightly higher than Young's modulus values measured from tensile curves [39]. Despite this eventual moderate shift, the use of this technique is still adapted since, in this study, what matters above all is the evolution of properties.

\section{Surface deformation on TNZ}

The preliminary study showed that deformation applied on the quenched state led to a decrease in elastic modulus. The gradient can thus be produced by surface deformation of a $\beta$ phase massive sample.

Plates were treated at $1173 \mathrm{~K}$ during $30 \mathrm{~min}$ under controlled atmosphere and water quenched. XRD analyses performed on surface and mid-thickness of control samples confirmed the presence of $\beta$ phase in the whole volume. Surface deformation was then carried out by shot-blasting, on a Wheelabrator industrial prototype (IRT-M2P Metz), using s130 steel balls at an intensity of 32A on Almen scale, which represents severe conditions. Static and dynamic tests were conducted.

As evidenced by Figure 3, shot-blasting of a $\beta$ quenched TNZ sample, under static condition at a coverage factor of $300 \%$, gave rise to an elasticity gradient. Instrumented indentation measurements revealed a decrease in modulus from about 85 to $65 \mathrm{GPa}$ over $400 \mu \mathrm{m}$ from the core to the surface of the sample. SEM observations showed an associated microstructural gradient, namely bands visible within a similar distance and whose density gradually decreases moving away from the surface. XRD highlighted the existence of martensite on surface of blasted samples.
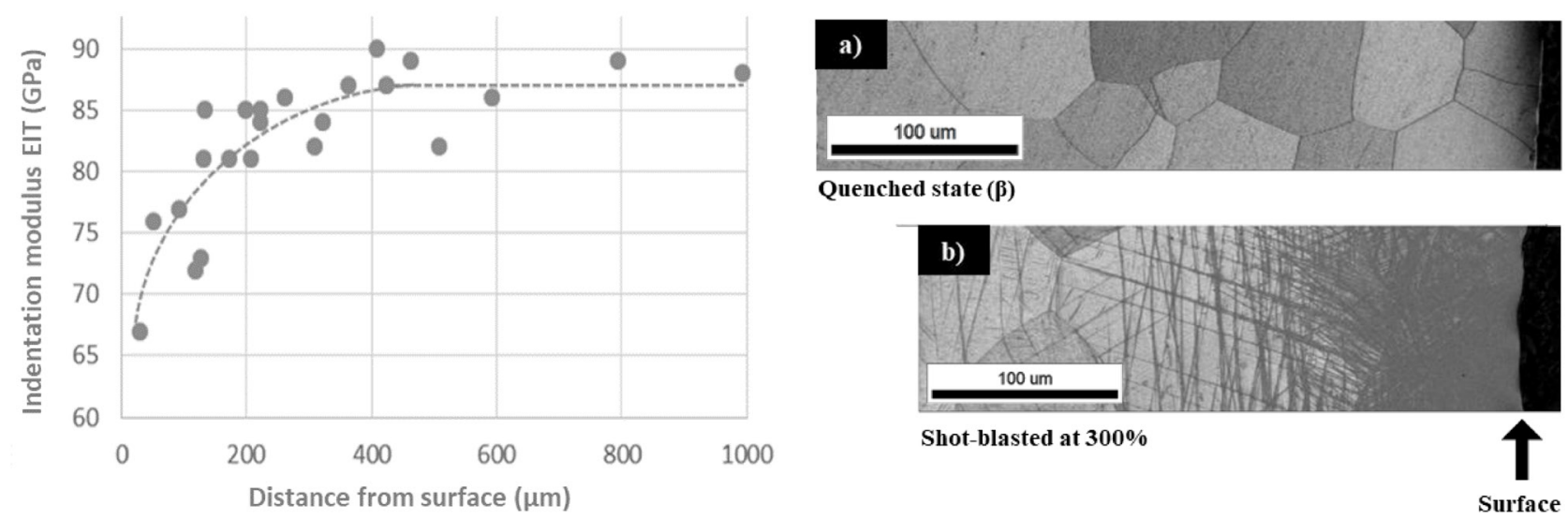

Figure 3 - Evolution of the indentation modulus (EIT) from the surface towards the core of a $\beta$ TNZ sample shot-blasted at $300 \%$ (left) and related microstructural observations by SEM, (a) before and (b) after shot-blasting (right).

\section{Preferential dissolution on Ti1313}

Determination of elastic range demonstrated that fine precipitation of an additional phase, $\alpha$ in the case of Ti-13-13, caused an increase in elastic modulus. Thinking about it in a reverse way, a gradient can therefore be created by precipitating $\alpha$ phase in the entire sample and then locally dissolve it only on surface.

For that purpose, plates were treated at $873 \mathrm{~K}$ during $1 \mathrm{~h}$ under controlled atmosphere and water quenched. Temperature was adjusted to obtain the same fine $\alpha+\beta$ microstructure as in the first part, despite a slightly different process. Plates were then cut into four lengthways to get samples with $5 \mathrm{~mm}$ square section. Dissolution was subsequently carried out by doing rapid thermaltreatments in molten carbonates bath. Various treating times and temperatures were investigated. However, no gradient was observed by this route; $\alpha$ phase being dissolved in the whole sample or not at all, even on surface. Making a parallel with precipitation phenomena, where cold-working can accelerate transformation kinetics [40], a supplementary driving force of mechanical origin was added. Under the same conditions as for TNZ, shot-blasting was thus performed on samples before flash thermal treatment to further facilitate the dissolution.

As Figure 4 illustrates, an elasticity gradient was made on an $\alpha+\beta$ Ti-13-13 sample shot-blasted during $15 \mathrm{~s}$ and then flashtreated $10 \mathrm{~s}$ at $1023 \mathrm{~K}$ in molten salts bath. From 130 to $75 \mathrm{GPa}$ over $100 \mu \mathrm{m}$, it represents a decrease in modulus of more than $40 \%$. Associated microstructural observations proved that, as desired, shot-blasting enabled to promote the dissolution of $\alpha$ phase on extreme surface. 


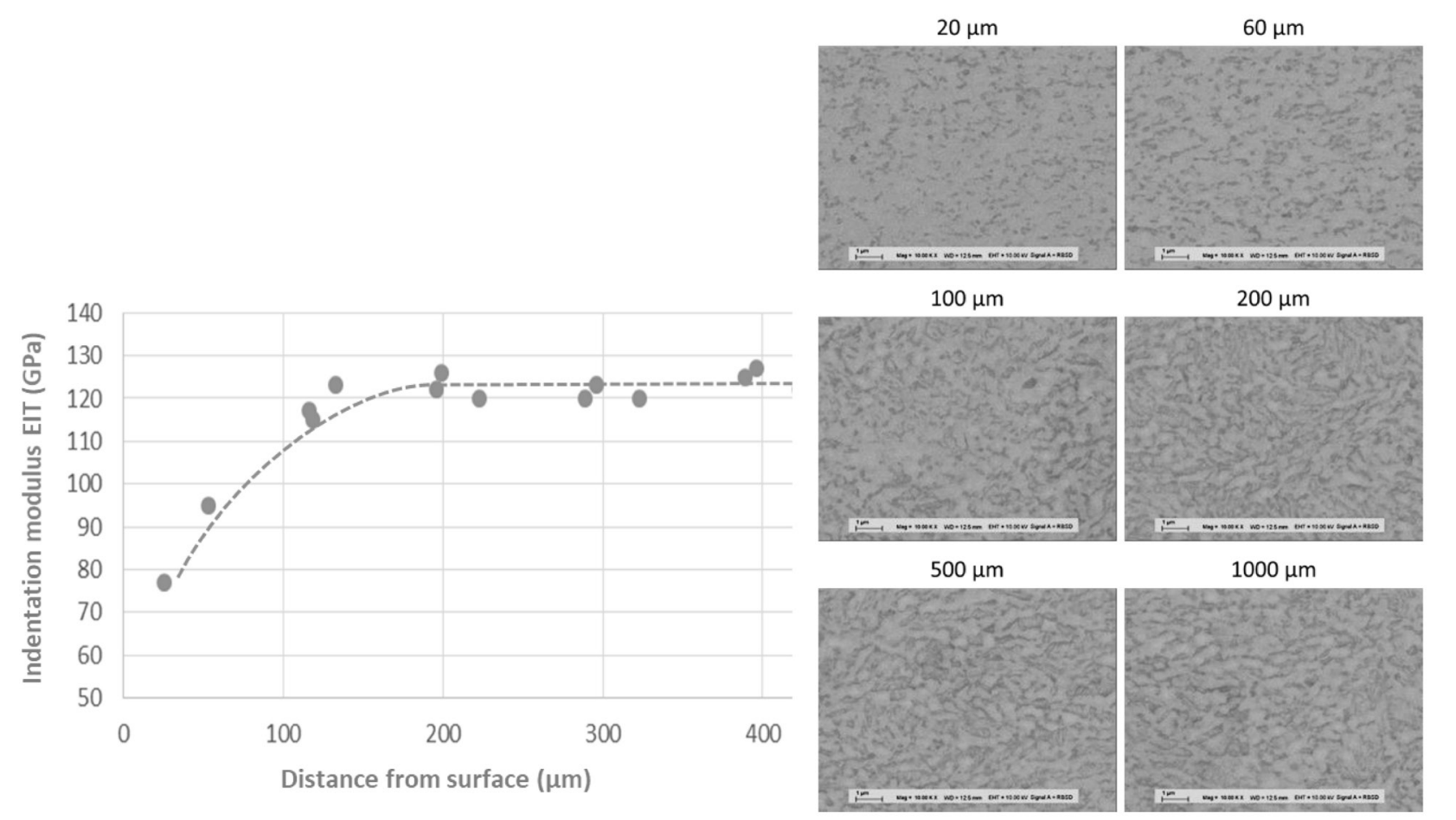

Figure 4 - Evolution of the indentation modulus from the surface towards the core of an $\alpha+\beta$ Ti-13-13 sample shot-blasted during 15 $s$ and then flash-treated $10 \mathrm{~s}$ at $1023 \mathrm{~K}$ (left), and related microstructural observations done by SEM in back scatter mode at various depth levels (right). $\alpha$ phase is dark and $\beta$ phase is light.

Although induced gradient could be optimized in terms of depth (for Ti-13-13) or modulus gap (for TNZ), maybe by looking for more adapted technical solutions to heat and deform, they nevertheless validate the bases material-strategy-process combinations which have been developed here. Furthermore, on each alloy, work can be done to apply both strategies, preferential dissolution then surface deformation, and thus amplify the elasticity gradient.

\section{Conclusions}

Original process routes based on appropriate combination of thermal and mechanical treatments, adapted to cautiously selected Ti$\mathrm{Nb}-\mathrm{Zr}$ alloys, have been effective in creating elastically graded materials. Indeed measurements performed by instrumented indentation revealed surface decrease in modulus from 85 to $65 \mathrm{GPa}$ over $400 \mu \mathrm{m}$ for TNZ alloy obtained by surface deformation, and from 130 to $75 \mathrm{GPa}$ over $100 \mu \mathrm{m}$ for Ti-13-13 by preferential dissolution.

This innovative solution allows to both maintain a high level of resistance in the main part of the piece and to reach a low modulus on surface. In other words, compared to previous technological options, this one enhances the strength-to-modulus ratio, $\sigma / E$, which can be perceived as a meaningful performance indicator for evaluating implantable biomaterials [41]. These promising results enable to look forward to the production of graded implanted devices which could promote osseointegration by reducing the elastic discontinuity at the bone / implant interface, and could have a profound impact in dental implantology but also in other fields such as orthopaedics.

\section{$\underline{\text { References }}$}

[1] D. F. Williams, Med. Device Technol., vol. 14, no. 8, pp. 10-13, 2003.

[2] P. Laheurte et al., Techniques de l'ingénieur (M4781). 2014.

[3] R. Rieger et al., J. Theor. Biol., vol. 274, no. 1, pp. 36-42, 2011. 
[4] A. Consolaro et al., Dent. Press J Orthod, vol. 15, no. 3, pp. 19-30, 2010.

[5] E. Kitamura et al., Clin. Oral Implants Res., vol. 15, pp. 401-412, 2004.

[6] M. Niinomi and M. Nakai, Int. J. Biomater, no. 836587, Jan. 2011.

[7] H. Kroger et al., Clinical orthopaedics and related research, no. 352. pp. 66-74, 1998.

[8] Z. Wally et al., Metals (Basel)., vol. 5, pp. 1902-1920, 2015.

[9] Y. Liu et al., Mater. Sci. Eng. C, vol. 49, pp. 323-329, 2015.

[10] S. J. Li et al., Acta Biomater., vol. 10, pp. 4537-4547, 2014.

[11] I. V. Okulov et al., Sci. Rep., vol. 7, no. 20, pp. 1-7, 2017.

[12] E. Koshy and S. R. Philip, Int J Clin Implant Dent, vol. 1, no. 1, pp. 14-22, 2015.

[13] A. Bandyopadhyay et al., Acta Biomater., vol. 6, no. 4, pp. 1640-1648, 2010.

[14] C. Oldani and A. Dominguez, in Recent Advances in Arthroplasty, InTech, 2012.

[15] Y. Oshida et al., Int. J. Mol. Sci., vol. 11, pp. 1580-1678, Jan. 2010.

[16] B. Piotrowski et al., Mater. Sci. Eng. C, vol. 38, pp. 151-160, May 2014.

[17] F. Prima and S. Nowak, WO 2012/059895 A1, 2012.

[18] C. C. Gomes et al., Genet. Mol. Biol., vol. 34, no. 1, pp. 116-21, Jan. 2011.

[19] S. G. Steinemann, in Evaluation of Biomaterials, WILEY-VCH, 1980, pp. 1-34.

[20] M. R. Riley et al., Toxicology, vol. 190, pp. 171-184, 2003.

[21] S. Rao et al., Biomed. Mater. Eng., vol. 6, pp. 79-86, 1996.

[22] M. Morinaga et al., in 6th World Conference on Titanium. III, 1988, pp. 1601-1606.

[23] M. Abdel-Hady Gepreel et al., Scr. Mater., vol. 55, no. 5, pp. 477-480, 2006.

[24] M. Tane et al., Acta Mater, vol. 56, no. 12, pp. 2856-2863, 2008.

[25] P. Laheurte et al., J. Mech. Behav. Biomed. Mater., vol. 3, no. 8, pp. 565-73, 2010.

[26] Y. L. Hao et al., Acta Biomater., vol. 3, no. 2, pp. 277-286, 2007.

[27] L. You and X. Song, Mater. Lett., vol. 80, pp. 165-167, 2012.

[28] M. Geetha et al., Prog. Mater. Sci., vol. 54, no. 3, pp. 397-425, 2009.

[29] Y. Zheng et al., Integr. Biomater. Biomed. Technol., pp. 253-272, 2012.

[30] Y. Li et al., Materials (Basel)., vol. 7, pp. 1709-1800, Mar. 2014.

[31] S. Miyazaki et al., Mater. Sci. Eng. A, vol. 438-440, pp. 18-24, Nov. 2006.

[32] M. Niinomi et al., Acta Biomater., vol. 8, no. 11, pp. 3888-3903, Nov. 2012.

[33] J. Y. Zhang et al., Mater. Sci. Eng. A, vol. 563, pp. 78-85, 2013.

[34] J. A. Davidson et al., Biomed. Mater. Eng., vol. 4, no. 3, pp. 231-243, 1994.

[35] M. Geetha et al., J. Alloys Compd., vol. 329, no. 1-2, pp. 264-271, Nov. 2001.

[36] P. Majumdar et al., J. Mech. Behav. Biomed. Mater., vol. 4, no. 7, pp. 1132-1144, Oct. 2011. 
[37] C. H. Park et al., Mater. Sci. Eng. A, vol. 527, no. 18-19, pp. 4914-4919, Jul. 2010.

[38] F. Sun et al., J. Mech. Behav. Biomed. Mater., vol. 4, no. 8, pp. 1864-1872, Nov. 2011.

[39] C. Fizanne-Michel, Thesis, 2014.

[40] V. Schulze, Modern Mechanical Surface Treatment, WILEY-VCH., vol. 53, no. 9. 2006.

[41] A. Biesiekierski et al., Nat. Sci. Reports, no. 8:5737, pp. 1-12, 2018. 